Commun. Korean Math. Soc. 26 (2011), No. 4, pp. 669-684

http://dx.doi.org/10.4134/CKMS.2011.26.4.669

\title{
THE LAW OF A STOCHASTIC INTEGRAL WITH TWO INDEPENDENT BIFRACTIONAL BROWNIAN MOTIONS
}

\author{
JUNFENG LiU
}

\begin{abstract}
In this note, we obtain the expression of the characteristic fucntion of the random variable $\int_{0}^{T} B_{s}^{\alpha, \beta} d B_{s}^{H, K}$, where $B^{\alpha, \beta}$ and $B^{H, K}$ are two independent bifractional Brownian motions with indices $\alpha \in(0,1), \beta \in(0,1]$ and $H K \in\left(\frac{1}{2}, 1\right)$, respectively.
\end{abstract}

\section{Introduction}

Given $H \in(0,1), K \in(0,1]$. The bifractional Brownian motion is a generalization of fractional brownian motion, defined as a centered self-similar Gaussian process $B^{H, K}=\left\{B_{t}^{H, K}, t \geq 0\right\}$ with indices $H$ and $K$ and covariance function

$$
E\left[B_{t}^{H, K} B_{s}^{H, K}\right]=\frac{1}{2^{K}}\left[\left(t^{2 H}+s^{2 H}\right)^{K}-|t-s|^{2 H K}\right], \quad \forall s, t \geq 0 .
$$

It is $H K$-self-similar and satisfies the following estimates

$$
2^{-K}|t-s|^{2 H K} \leq E\left[\left(B_{t}^{H, K}-B_{s}^{H, K}\right)^{2}\right] \leq 2^{1-K}|t-s|^{2 H K} .
$$

Clearly, if $K=1$, the process is a fractional Brownian motion with Hurst parameter $H$. This process was first introduced by Houdré and Villa [7]. Russo and Tudor [16] have established some properties on the strong variations, local times and stochastic calculus of real-valued bifractional Brownian motion. An interesting property that deserves to be recalled is the fact that, when $H K=$ $\frac{1}{2}$, the quadratic variation of this process on $[0, t]$ is equal to $2^{1-K} t$. TudorXiao [17] studied some sample path properties of bifractional Brownian motion. Itô's formula and Tanaka formula for multidimensional bifractional Brownian motion were given by Es-sebaiy and Tudor [6].

Clearly $B^{H, K}$ is neither a Markov process nor a semimartingale unless $H=\frac{1}{2}$ and $K=1$, so many of the powerful techniques from stochastic analysis are not

Received June 12, 2010; Revised February 12, 2011.

2010 Mathematics Subject Classification. 60H05, 60H07.

Key words and phrases. bifractional Brownian motion, stochastic integral, characteristic function.

The research is supported by NSFC (10871041) and NSRC (10023). 
available when dealing with $B^{H, K}$. However it has some properties analogous to those of fractional Brownian motion, but its increments are not stationary. In this note we consider the law of the stochastic integral

$$
\int_{0}^{T} B_{s}^{\alpha, \beta} d B_{s}^{H, K}
$$

where $B^{\alpha, \beta}$ and $B^{H, K}$ are two independent bi-fBm with $\alpha \in(0,1), \beta \in(0,1]$ and $2 H K>1$.

We have known that it is difficult to compute the law of a stochastic integral with respect to the Wiener process when the integrand is not deterministic. The systematic study for this problem was initiated in Lévy [12]. He showed that the characteristic function of $A_{t}=\int_{0}^{t} X_{s} d Y_{s}-\int_{0}^{t} Y_{s} d X_{s}$ is

$$
E\left(e^{i u A_{t}}\right)=\frac{1}{\cosh (u t)}, \quad t \geq 0, u \in \mathbb{R},
$$

where $\left(X_{t}, Y_{t}\right)$ is an $\mathbb{R}^{2}$-valued Brownian motion with $\left(X_{0}, Y_{0}\right)=(0,0)$. This is called Lévy's stochastic area formula. Berthuet [3] and Yor [19] (see also Protter $[15])$ gave the other proof, and considered the law of the random variables

$$
\lambda \int_{0}^{t} X_{s} d Y_{s}+\rho \int_{0}^{t} Y_{s} d X_{s}, \quad t \geq 0 .
$$

Moreover the density function for the distribution of $A(t)$ is

$$
f_{A(t)}(x)=\frac{1}{2 t \cosh (\pi x / 2 t)}, \quad x \in \mathbb{R} .
$$

The stochastic area process $A$ shares some of properties of Brownian motion. For example $A$ satisfies a reflection principle. If one changes the sign of the increments of $A$ after a stopping time, the process obtained thereby has the same distribution as that of $A$. One can use this fact to show, for example, that if $S_{t}=\sup _{0 \leq s \leq t} A(s)$, then $S_{t}$ has the same distribution as $|A(t)|$ for $t>0$.

As an extension, recently, Bardina-Tudor [2] considered a similar integral driven by fractional Brownian motions, and they obtained the characteristic function of the random variable $S=\int_{0}^{1} B_{s}^{\alpha} d B_{s}^{H}$, where $B^{\alpha}$ and $B^{H}$ are two independent fractional Brownian motions with Hurst indexes $\alpha \in(0,1)$ and $H \in\left(\frac{1}{2}, 1\right)$, respectively. As well-known, in recent years, the long-range dependence have attracted much attention, both for their mathematical interest and their applications in stochastic control in telecommunication, turbulence, finance and etc. The best known and most-used process that exhibits longrange dependence is the fractional Brownian motion ( $\mathrm{fBm}$ for short). These due to its interesting properties and its applications in various scientific areas including telecommunications, turbulence, image processing and finance. The self-similarity and stationarity of the increments are two main properties for which fractional Brownian motion enjoyed success as a modeling tool. The fractional Brownian motion is the only continuous Gaussian process which is 
self-similar and has stationary increments. Some surveys and complete literatures could be found in Alós et al. [1], Biagini et al. [4], Hu [8], Mishura [13], Nualart [14]. However, contrast to the extensive studies on fractional Brownian motion, there has been little systematic investigation on other self-similar Gaussian processes. The main reasons for this are the complexity of dependence structures and the non-availability of convenient stochastic integral representations for self-similar Gaussian processes which do not have stationary increments. On the other hand, many authors have proposed to use more general self-similar Gaussian processes and random fields as stochastic models, and such applications have raised many interesting theoretical questions about self-similar Gaussian processes and fields in general. Thus, it seems interesting to study the law of stochastic integrals driven by more general self-similar Gaussian processes.

This note is organized as follows. In Section 2 we present some preliminaries for bi-fBm and the Wiener integral with respect to bi-fBm. In Section 3 we obtained the characteristic function of stochastic integral $\int_{0}^{T} B_{t}^{\alpha, \beta} d B_{t}^{H, K}$. The case of two-parameter is considered in Section 4.

\section{Preliminaries on bifBm and Wiener integral}

In this section, we briefly recall some basic definitions and results of bifractional Brownian motion. As we have pointed out before, bifractional Brownian motion (bifBm in short) $B^{H, K}=\left\{B_{t}^{H, K}, 0 \leq t \leq T\right\}$, on the probability space $(\Omega, \mathscr{F}, P)$ with indices $H \in(0,1)$ and $K \in(0,1]$ is a rather special class of self-similar Gaussian processes such that $B_{0}^{H, K}=0$ and

$$
E\left[B_{t}^{H, K} B_{s}^{H, K}\right]=R_{H, K}(t, s):=\frac{1}{2^{K}}\left[\left(t^{2 H}+s^{2 H}\right)^{K}-|t-s|^{2 H K}\right], \quad \forall s, t \geq 0 .
$$

The process was first introduced by Houdré and Villa in [7] and a stochastic calculus can be found in [17]. It is $H K$-self similar and satisfies the following estimates (the quasi-helix property in the sense of R. Klein et al. [9])

$$
2^{-K}|t-s|^{2 H K} \leq E\left[\left(B_{t}^{H, K}-B_{s}^{H, K}\right)^{2}\right] \leq 2^{1-K}|t-s|^{2 H K} .
$$

Thus, Kolmogorov's continuity criterion implies that bifractional Brownian motion is Hölder continuous of order $\delta$ strictly less than $H K$. An interesting property is that the quadratic covariation of this process on $[0, t]$ equals to $2^{1-K} t$ provided $2 H K=1$. Recently, Lei-Nualart [11] have shown a decomposition of the bifractional Brownian motion with parameters $H$ and $K$ into the sum of a fractional Brownian motion with Hurst index $H K$ plus a Gaussian process with absolutely continuous trajectories. Russo-Tudor [16] showed that the bifractional Brownian motion behaves as a fractional Brownian motion with Hurst index $H K$. The stochastic calculus with respect to the bifractional Brownian motion has been recently developed by Kruk-Russo-Tudor [10] by 
combining Mallavin Calculus and a notion of Covariance Measure Structure. More works on bifractioal Brownian motion can be found in Tudor-Xiao [17], Es-sabaiy-Tudor [6], Yan et al. [18] and the references therein.

Consider $\mathscr{E}$ the class of step functions of the form

$$
f=\sum_{j=1}^{n} a_{j} 1_{\left[t_{j}-t_{j-1}\right)}, \quad 0=t_{0}<t_{1}<t_{2}<\cdots<t_{n}=T, a_{j} \in \mathbb{R} .
$$

We denote by $\mathcal{H}_{H, K}$ the canonical space of the bifractional Brownian motion. That is $\mathcal{H}_{H, K}$ is the completion of the linear space $\mathscr{E}$ generated by the indicator functions $\left\{1_{[0, t]}, t \in[0, T]\right\}$ with respect to the inner product

$$
\left\langle 1_{[0, s]}, 1_{[0, t]}\right\rangle_{\mathcal{H}_{H, K}}=R_{H, K}(s, t) .
$$

$\mathcal{H}_{H, K}$ now is a Hilbert space equipped with the scalar product $\left\langle 1_{[0, s]}, 1_{[0, t]}\right\rangle_{\mathcal{H}_{H, K}}$. The structure of the Hilbert space $\mathcal{H}_{H, K}$ varies upon the values of $H$ and $K$. As usual, we can define the linear application

$$
\Phi: \mathscr{E} \rightarrow L^{2}(\Omega, \mathcal{F}, P)
$$

by

$$
1_{[0, t]} \mapsto \Phi\left(1_{[0, t]}\right)=\int_{0}^{T} 1_{[0, t]}(s) d B_{s}^{H, K} \equiv B_{t}^{H, K} .
$$

The application can be extended to a linear isometry between $\mathcal{H}_{H, K}$ and the Gaussian space associated with $B^{H, K}$. We will denote the isometry by $\varphi \rightarrow$ $B^{H, K}(\varphi)$ and let

$$
\begin{aligned}
\phi_{H, K}(s, t)=\frac{2 H K}{2^{K}}[ & 2 H(K-1)\left(t^{2 H}+s^{2 H}\right)^{K-2} t^{2 H-1} s^{2 H-1} \\
+ & \left.(2 H K-1)|t-s|^{2 H K-2}\right] .
\end{aligned}
$$

From Yan et al. [18], we know that there exist two positive constants $C_{H, K}$ and $c_{H, K}$ such that

$$
c_{H, K}|t-s|^{2 H K-2} \leq\left|\phi^{H, K}(s, t)\right| \leq C_{H, K}|t-s|^{2 H K-2} .
$$

We call $B^{H, K}(\varphi):=\int_{0}^{T} \varphi(t) d B_{t}^{H, K}$ the Wiener integral of $\varphi$ with respect to $B_{t}^{H, K}$.

However working with the space $\mathcal{H}_{H, K}$ is not convenient. First, because it may also contain distribution (in the case of fractional Brownian motion when $K=1$ ) and second, because the norm in this space is not always tractable. We will use the subspace $|\mathcal{H}|_{H, K}$ of $\mathcal{H}_{H, K}$ which is defined as the set of measurable function $f$ on the interval $[0, T]$ with

$$
\|f\|_{|\mathcal{H}|_{H, K}}^{2}:=\int_{0}^{T} \int_{0}^{T}|f(s)||f(r)|\left|\phi_{H, K}(s, r)\right| d r d s<\infty .
$$

From Kruk et al. [10], we know the space $|\mathcal{H}|_{H, K}$ is a Banach space with respect to the norm $\|\cdot\|_{|\mathcal{H}|_{H, K}}$ and it is included in the space $\mathcal{H}_{H, K}$. 
It has been proved in Kurk et al. [10] that $\mathcal{E}$ is dense in $\mathcal{H}_{H, K}$. For $\frac{1}{2}<$ $H K<1$ we denote by $\mathcal{S}$ the set of smooth functionals of the form

$$
F=f\left(B^{H, K}\left(\varphi_{1}\right), \ldots, B^{H, K}\left(\varphi_{n}\right)\right),
$$

where $f \in C_{b}^{\infty}\left(\mathbb{R}^{n}\right)$ and $\varphi_{i} \in \mathcal{H}_{H, K}$. The Malliavin derivative of a functional $F$ as above is given by

$$
D^{H} F=\sum_{i=1}^{n} \frac{\partial f}{\partial x_{i}}\left(B^{H, K}\left(\varphi_{1}\right), \ldots, B^{H, K}\left(\varphi_{n}\right)\right) \varphi_{i},
$$

and this operator can be extended to the closure $\mathbb{D}^{m, 2}(m \geq 1)$ of $\mathcal{S}$ with respect to the norm

$$
\|F\|_{m, 2}^{2} \equiv E|F|^{2}+E\left\|D^{H, K} F\right\|_{\mathcal{H}_{H, K}}^{2}+\cdots+E\left\|D^{H, K, m} F\right\|_{\mathcal{H}_{H, K}^{\widehat{\otimes}, m}}^{2},
$$

where $\mathcal{H}_{H, K}^{\widehat{\otimes} m}$ denotes the $m$ fold symmetric tensor product and the $m$-th derivative $D^{H, K, m}$ is defined by iteration. The divergence integral $\delta^{H, K}$ is the adjoint operator of $D^{H, K}$. Concretely, a random variable $u \in L^{2}\left(\Omega, \mathcal{H}_{H, K}\right)$ belongs to the domain of the divergence operator $\delta^{H, K}\left(\right.$ in symbol $\operatorname{Dom}\left(\delta^{H}\right)$ ) if

$$
E\left|\left\langle D^{H, K} F, u\right\rangle_{\mathcal{H}_{H, K}}\right| \leq c\|F\|_{L^{2}(\Omega)}
$$

for every $F \in \mathcal{S}$. In this case $\delta^{H, K}(u)$ is given by the duality relationship

$$
E\left(F \delta^{H, K}(u)\right)=E\left\langle D^{H, K} F, u\right\rangle_{\mathcal{H}_{H, K}}
$$

for any $F \in \mathbb{D}^{1,2}$, and we have the following integration by parts:

$$
F \delta^{H, K}(u)=\delta^{H, K}(F u)+\left\langle D^{H, K} F, u\right\rangle_{\mathcal{H}_{H, K}}
$$

for any $u \in \operatorname{Dom}\left(\delta^{H}\right), F \in \mathbb{D}^{1,2}$ such that $F u \in L^{2}\left(\Omega, \mathcal{H}_{H, K}\right)$. It follows that

$$
E\left[\delta^{H, K}(u)^{2}\right]=E\|u\|_{\mathcal{H}_{H, K}}^{2}+E\left\langle D^{H} u,\left(D^{H, K} u\right)^{*}\right\rangle_{\mathcal{H}_{H, K} \otimes \mathcal{H}_{H, K}},
$$

where $\left(D^{H, K} u\right)^{*}$ is the adjoint of $D^{H, K} u$ in the Hilbert space $\mathcal{H}_{H, K} \otimes \mathcal{H}_{H, K}$. We also will use the notation

$$
\delta^{H, K}(u)=\int_{0}^{T} u_{s} d B_{s}^{H, K},
$$

to express the Skorohod integral of an adapted process $u$. It is also possible to introduce multiple integrals $I_{n}\left(f_{n}\right), f_{n} \in \mathcal{H}_{H, K}^{\otimes n}$ with respect to $B^{H, K}$. For the divergence integral we have the following convergence: if $\left\{u_{n}\right\}$ is a sequence of elements in $\operatorname{Dom}\left(\delta^{H, K}\right)$ such that $u_{n} \rightarrow u$ in $L^{2}\left(\Omega ; \mathcal{H}_{H, K}\right)$, and $\delta^{H, K}\left(u_{n}\right) \rightarrow G$ in $L^{2}(\Omega)$, then we have $u \in \operatorname{Dom}\left(\delta^{H}\right)$ and $\delta^{H, K}(u)=G$.

Clearly, for any $\varphi \in \mathcal{H}_{H, K}$ the the Wiener integral with respect to $B^{H, K}$ can be defined as

$$
\int_{0}^{T} \varphi(s) d B_{s}^{H, K}=\lim _{n \rightarrow \infty} \sum_{j=1}^{n} \varphi\left(s_{j}\right)\left(B_{s_{j}}^{H, K}-B_{s_{j-1}}^{H, K}\right),
$$


where $\left\{0=s_{0}, s_{1}, \ldots, s_{n}=T\right\}$ is a partition of $[0, T]$ such that $\max _{i}\left\{\left|s_{i+1}-s_{i}\right|\right\} \rightarrow$ 0 , as $n$ tends to infinity. In fact, denote $I_{n}=\sum_{j=1}^{n} \varphi\left(s_{j}\right)\left(B_{s_{j}}^{H, K}-B_{s_{j-1}}^{H, K}\right)$, according to (2.8), we have

$$
\begin{aligned}
I_{n} & =\sum_{j=1}^{n} \varphi\left(s_{j}\right)\left(B_{s_{j}}^{H, K}-B_{s_{j-1}}^{H, K}\right)=\sum_{j=1}^{n} \varphi\left(s_{j}\right) \delta^{H, K}\left(\mathbf{1}_{\left(s_{j-1}, s_{j}\right]}(\cdot)\right), \\
& =\sum_{j=1}^{n} \delta^{H, K}\left(\varphi\left(s_{j}\right) \mathbf{1}_{\left(s_{j-1}, s_{j}\right]}(\cdot)\right)=\delta^{H, K}\left(\sum_{j=1}^{n} \varphi\left(s_{j}\right) \mathbf{1}_{\left(s_{j-1}, s_{j}\right]}(\cdot)\right),
\end{aligned}
$$

since the Malliavin derivative of $\varphi\left(s_{j}\right)$ is zero. From the proof of Theorem 1 in K. Es-sebaiy et al. [6], for a function $f \in C^{2}$ on $\mathbb{R}$ satisfying some regularity condition, we know that the term

$$
I_{1}^{n}=\delta^{H, K}\left(\sum_{j=1}^{n} f^{\prime}\left(B_{t_{j-1}}^{H, K}\right) \mathbf{1}_{\left(s_{j-1}, s_{j}\right]}(\cdot)\right)
$$

converges to $\delta^{H, K}\left(f^{\prime}\left(B^{H, K}\right)\right)$ in $L^{2}(\Omega)$. Moreover, for

$$
\varphi \in L_{\mu}=\left\{\varphi: \int_{0}^{T} \int_{0}^{T} \varphi(s) \varphi(t) \mu(d s, d t)<\infty\right\}
$$

where $\mu$ is a positive measure, then Proposition 6.6 in I. Kruk et al [10] implies that

(Wiener integral) $\quad \int_{0}^{T} \varphi(s) d X_{s}=\int_{0}^{T} \varphi(s) \delta X_{s}, \quad$ (Divergence integral)

where $X$ is a Gaussian process. That is to say, when the integrand is deterministic, the Wiener integral with respect to a Gaussian process coincides with the divergence integral with respect to it. Combining the above two results and choosing $X=B^{H, K}$ and $\mu(d s, d t)=\left|\phi_{H, K}(s, t)\right| d s d t$, we can obtain the convergence (2.9)

Moreover, if stochastic process $u$ is independent of $B^{H, K}$, then the Skorohod integral $\int_{0}^{T} u(s) d B_{s}^{H, K}$ can be defined as (2.9) since the Malliavin derivative of $u$ is zero.

More generally, for $H K \in\left(\frac{1}{2}, 1\right)$. Let $|\mathcal{H}|_{H, K}^{n}$ be the set of functions $f$ : $T^{n} \mapsto \mathbb{R}$ with

$$
\begin{aligned}
&\|f\|_{|\mathcal{H}|_{H, K}^{n}=}=\int_{[0, T]^{2 n}} \mid f\left(u_{1}, \ldots u_{n}\right)|| f\left(v_{1}, \ldots v_{n}\right) \mid \\
& \cdot\left(\prod_{i=1}^{n}\left|\phi_{H, K}\left(u_{i}, v_{i}\right)\right|\right) d u_{1} \cdots d u_{n} d v_{1} \cdots d v_{n}<\infty .
\end{aligned}
$$

We will denote by $|\mathcal{H}|_{H, K, S}^{n}$ the set of symmetric functions $f \in|\mathcal{H}|_{H, K}^{n}$. For $f \in|\mathcal{H}|_{H, K, S}^{2}$, according to P. Caithamer [5], define the operator $K_{f}^{H, K}$ : 
$|\mathcal{H}|_{H, K, S}^{2} \rightarrow|\mathcal{H}|_{H, K, S}^{2}$

$$
\left(K_{f}^{H, K} \varphi\right)(y)=\int_{0}^{T} \int_{0}^{T} f(x, y) \varphi\left(x^{\prime}\right)\left|\phi_{H, K}\left(x, x^{\prime}\right)\right| d x d x^{\prime} .
$$

One can easily check that if $f$ is positive and $H K \in\left(\frac{1}{2}, 1\right)$, then the eigenvalues of the operator $K_{f}^{H, K}$ are positive. In fact, we can write

$$
\left(K_{f}^{H, K} \psi\right)(y)=\int_{0}^{T} A\left(x^{\prime}, y\right) \psi\left(x^{\prime}\right) d x^{\prime},
$$

where $A\left(x^{\prime}, y\right)=\int_{0}^{T} f(x, y)\left|\phi_{H, K}\left(x, x^{\prime}\right)\right| d x$ is positive, then the operator $K_{f}^{H, K}$ is a positive operator. It is noteworthiness that the operator $K_{f}^{H, K}$ will be changed as

$$
K_{f}^{H, K} \varphi(y)=\int_{0}^{T} f(x, y) \varphi(x) d x
$$

provided $H K=\frac{1}{2}$.

\section{The characteristic function of the double integral}

Throughout this section, $B^{H, K}$ and $B^{\alpha, \beta}$ will denote two independent bifractional Brownian motions with indices $H, K$ and $\alpha, \beta$ respectively. We'll compute the characteristic function of the random variable

$$
\ell:=\int_{0}^{T} B_{s}^{\alpha, \beta} d B_{s}^{H, K} .
$$

In the following, we need to restrict ourselves to the situation $H K \in\left(\frac{1}{2}, 1\right)$, then the random variable $\ell$ of the form (3.1) is well-defined.

We start with the following lemma which gives an approximation of the random variable $\ell$ given by (3.1) when $H K>\frac{1}{2}$.

Lemma 3.1. Assume $H K>\frac{1}{2}$ and $\alpha, \beta \in(0,1)$. Denote by

$$
T_{n}=\sum_{i=0}^{n-1} B_{t_{i}}^{\alpha, \beta}\left(B_{t_{i+1}}^{H, K}-B_{t_{i}}^{H, K}\right),
$$

where $\pi: 0=t_{0}<t_{1}<t_{2}<\cdots<t_{n}=T$ denotes a partition of $[0,1]$. Then it holds that

$$
T_{n} \rightarrow \ell,
$$

in $L^{2}(\Omega)$ as as $|\Delta|_{n}=\max _{n \geq 1}\left|t_{n}-t_{n-1}\right|$ tends to zero.

Proof. Because of the independence of $B^{H, K}$ and $B^{\alpha, \beta}$, we can write

$$
B_{t_{i}}^{\alpha, \beta}\left(B_{t_{i+1}}^{H, K}-B_{t_{i}}^{H, K}\right)=\int_{t_{i}}^{t_{i+1}} B_{t_{i}}^{\alpha, \beta} d B_{s}^{H, K} .
$$


It suffices to show that

$$
\sum_{i=0}^{n-1} B_{t_{i}}^{\alpha, \beta} 1_{\left[t_{i}, t_{i+1}\right)}(\cdot) \rightarrow B^{\alpha, \beta}=\sum_{i=0}^{n-1} B^{\alpha, \beta} 1_{\left[t_{i}, t_{i+1}\right)}(\cdot),
$$

in $L^{2}(\Omega) \times|\mathcal{H}|_{H, K}$ as $|\Delta|_{n} \rightarrow 0$. In fact, due to the independence of the two bifractional Brownian motions. Using formula (2.11), we have

$$
\begin{aligned}
& \mathrm{E}\left\|\sum_{i=0}^{n-1}\left(B_{t_{i}}^{\alpha, \beta}-B_{\cdot}^{\alpha, \beta}\right) 1_{\left[t_{i}, t_{i+1}\right]}\right\|_{|\mathcal{H}|_{H, K}}^{2} \\
= & \sum_{i, j=0}^{n-1} \int_{t_{i}}^{t_{i+1}} \int_{t_{j}}^{t_{j+1}} \mathrm{E}\left(B_{t_{i}}^{\alpha, \beta}-B_{s}^{\alpha, \beta}\right)\left(B_{t_{j}}^{\alpha, \beta}-B_{r}^{\alpha, \beta}\right) \phi(r, s) d r d s \\
\leq & \sum_{i, j=0}^{n-1} \int_{t_{i}}^{t_{i+1}} \int_{t_{j}}^{t_{j+1}}\left(\mathrm{E}\left(B_{t_{i}}^{\alpha, \beta}-B_{s}^{\alpha, \beta}\right)^{2}\right)^{\frac{1}{2}}\left(\mathrm{E}\left(B_{t_{j}}^{\alpha, \beta}-B_{r}^{\alpha, \beta}\right)^{2}\right)^{\frac{1}{2}}|\phi(r, s)| d r d s \\
\leq & 2^{2-\beta-K} C_{H K} \sum_{i, j=0}^{n-1} \int_{t_{i}}^{t_{i+1}} \int_{t_{j}}^{t_{j+1}}\left|t_{i}-s\right|^{\alpha \beta}\left|t_{j}-r\right|^{\alpha \beta}|s-r|^{2 H K-2} d r d s \\
\leq & 2^{2-\beta-K} C_{H K}|\Delta|_{n}^{2 \alpha \beta} \sum_{i, j=0}^{n-1} \int_{t_{i}}^{t_{i+1}} \int_{t_{j}}^{t_{j+1}}|s-r|^{2 H K-2} d r d s
\end{aligned}
$$

and this tends to zero for every $\alpha, \beta \in(0,1)$ as $|\Delta|_{n} \rightarrow 0$.

We will also need the following technical lemma.

Lemma 3.2. i) Assume $\alpha \beta \in\left(\frac{1}{2}, 1\right)$ and consider the function

$$
\begin{aligned}
& f^{H, K}(x, y) \\
= & T^{2 H K}-\frac{1}{2^{K}}\left\{\left(T^{2 H}+x^{2 H}\right)^{K}+\left(T^{2 H}+y^{2 H}\right)^{K}-(T-x)^{2 H K}\right. \\
& \left.\quad-(T-y)^{2 H K}-\left(x^{2 H}+y^{2 H}\right)^{K}+|x-y|^{2 H K}\right\},
\end{aligned}
$$

where $x, y \in[0, T]$. Then $f^{H, K} \in|\mathcal{H}|_{\alpha, \beta, S}^{2}$.

ii) Assume $H K \in\left(\frac{1}{2}, 1\right)$ and consider the function

$$
f^{\alpha, \beta}(x, y)=\frac{1}{2^{\beta}}\left[\left(x^{2 \alpha}+y^{2 \alpha}\right)^{\beta}-|x-y|^{2 \alpha \beta}\right],
$$

where $x, y \in[0, T]$. Then $f^{\alpha, \beta} \in|\mathcal{H}|_{H, K, S}^{2}$.

Proof. At first let's prove the point i); the point ii) is similar. We have to show that

$$
\begin{aligned}
I:= & \int_{[0, T]^{4}}\left|f^{H, K}\left(x_{1}, y_{1}\right)\right|\left|f^{H, K}\left(x_{2}, y_{2}\right)\right|\left|\phi_{\alpha, \beta}\left(x_{1}, x_{2}\right)\right| \\
& \left|\phi_{\alpha, \beta}\left(y_{1}, y_{2}\right)\right| d x_{1} d x_{2} d y_{1} d y_{2}<\infty .
\end{aligned}
$$


Note that

$$
\begin{aligned}
\left|f^{H, K}\left(x_{i}, y_{i}\right)\right| & =\mathrm{E}\left(B_{T}^{H, K}-B_{x_{i}}^{H, K}\right)\left(B_{T}^{H, K}-B_{y_{i}}^{H, K}\right) \\
& \leq\left(\mathrm{E}\left(B_{T}^{H, K}-B_{x_{i}}^{H, K}\right)^{2}\right)^{\frac{1}{2}}\left(\mathrm{E}\left(B_{T}^{H, K}-B_{y_{i}}^{H, K}\right)^{2}\right)^{\frac{1}{2}} \\
& =2^{1-K}\left(T-x_{i}\right)^{H K}\left(T-y_{i}\right)^{H K}
\end{aligned}
$$

Then the integral $I$ is therefore bounded by

$$
\begin{aligned}
& I \leq 2^{2-2 K} C_{\alpha, \beta} \int_{[0, T]^{4}}\left|T-x_{1}\right|^{H K}\left|T-y_{1}\right|^{H K}\left|T-x_{2}\right|^{H K}\left|T-y_{2}\right|^{H K} \\
& \cdot\left|x_{1}-x_{2}\right|^{2 \alpha \beta-2}\left|y_{1}-y_{2}\right|^{2 \alpha \beta-2} d x_{1} d x_{2} d y_{1} d y_{1} \\
&=\left(2^{1-K} C_{\alpha, \beta} \int_{0}^{T} \int_{0}^{T}\left(T-x_{1}\right)^{H K}\left(T-x_{2}\right)^{H K}\left|x_{1}-x_{2}\right|^{2 \alpha \beta-2} d x_{1} d x_{2}\right)^{2} .
\end{aligned}
$$

Now using the change of variable $z=\frac{x-y}{T-y}$, we get

$$
\begin{aligned}
I_{1}: & =\int_{[0, T]^{2}}(T-x)^{H K}(T-y)^{H K}|x-y|^{2 \alpha \beta-2} d y d x \\
& =2 \int_{0}^{T} \int_{0}^{x}(T-x)^{H K}(T-y)^{H K}|x-y|^{2 \alpha \beta-2} d y d x \\
& =2 \int_{0}^{T} \int_{0}^{\frac{x}{T}}(T-x)^{2 H K+2 \alpha \beta-1}(1-z)^{-H K-2 \alpha \beta} z^{2 \alpha \beta-2} d z d x \\
& =2 \int_{0}^{1}(1-z)^{-H K-2 \alpha \beta} z^{2 \alpha \beta-2}\left(\int_{T z}^{T}(T-x)^{2 H K+2 \alpha \beta-1} d x\right) d z \\
& =\frac{1}{H K+\alpha \beta} \int_{0}^{1}(1-z)^{-H K-2 \alpha \beta} z^{2 \alpha \beta-2}(T-T z)^{2 H K+2 \alpha \beta} d z \\
& =\frac{T^{2 H K+2 \alpha \beta}}{H K+\alpha \beta} B(2 \alpha \beta-1, H K+1)<\infty,
\end{aligned}
$$

using that $\alpha \beta \in\left(\frac{1}{2}, 1\right)$ and $B(a, b)$ denotes the Beta function. Then we complete the proof.

We state now our main result of this paper. The point ii) allows us to consider the situation when the parameters of the integrator $\alpha \beta<\frac{1}{2}$.

Theorem 3.1. Let $\alpha, \beta \in\left(\frac{1}{2}, 1\right)$ and $H K \in\left(\frac{1}{2}, 1\right)$. Then the characteristic function of the random variable $\ell$ given by (3.1) is

$$
\mathrm{E} e^{i t \ell}=\prod_{i \geq 1}\left(1+\frac{1}{t^{2} \mu_{i}}\right)^{\frac{1}{2}}
$$


where $\left(\mu_{i}\right)_{i \geq 1}$ are the eigenvalues of the operator $K_{f^{H, K}}^{\alpha, \beta}$ given by (2.12) and $f^{H, K}$ is defined by (3.3).

Proof. By Lemma 3.1, we have

$$
\mathrm{E} e^{i t \ell}=\lim _{n \rightarrow \infty} \mathrm{E} e^{i t T_{n}}
$$

where $T_{n}$ is given by (3.2) with $t_{i}=\frac{i}{n} T$ for every $i=0,1, \ldots, n-1$. Let us compute the characteristic function of the random variable $T_{n}$. We will using the following fact: If $X, Y$ are two independent random variables, then

$$
\mathrm{E}[\Phi(X, Y) \mid X]=\varphi(X)
$$

where $\varphi(x)=\mathrm{E}[\Phi(x, Y)]$.

Let us put

(3.6) $X=\left(B_{0}^{\alpha, \beta}, B_{\frac{1}{n} T}^{\alpha, \beta}, \ldots B_{\frac{n-1}{n} T}^{\alpha, \beta}\right), Y=\left(B_{\frac{1}{n} T}^{H, K}-B_{0}^{H, K}, \ldots, B_{\frac{n}{n} T}^{H, K}-B_{\frac{n-1}{n} T}^{H, K}\right)$.

Therefore we obtain

$$
\varphi(x)=\mathrm{E}\left(e^{i t \sum_{k=0}^{n-1} x_{k} Y_{k}}\right)=e^{-\frac{t^{2}}{2} x^{T} A^{H, K} x},
$$

where the matrix $A^{H, K}=\left(A_{k, l}^{H, K}\right)_{k, l=0,1, \ldots, n-1}$ is given by

$$
\begin{aligned}
& A_{k, l}^{H, K} \\
= & \mathrm{E}\left(B_{\frac{k+1}{n} T}^{H, K}-B_{\frac{k}{n} T}^{H, K}\right)\left(B_{\frac{l+1}{n} T}^{H, K}-B_{\frac{l}{n} T}^{H, K}\right) \\
= & \frac{1}{2^{K}}\left(\frac{T}{n}\right)^{2 H K}\left[\left((k+1)^{2 H}+(l+1)^{2 H}\right)^{K}-\left(k^{2 H}+(l+1)^{2 H}\right)^{K}\right. \\
& -\left((k+1)^{2 H}+l^{2 H}\right)^{K}+\left(k^{2 H}+l^{2 H}\right)^{K}+|k-1-l|^{2 H K}+|k-l+1|^{2 H K} \\
& \left.-2|k-l|^{2 H K}\right] \\
= & \frac{1}{2^{K}}\left(\frac{T}{n}\right)^{2 H K}\left(f_{1}(k, l)+f_{2}(k, l)\right),
\end{aligned}
$$

where

$$
\begin{aligned}
f_{1}(k, l)= & \left((k+1)^{2 H}+(l+1)^{2 H}\right)^{K}-\left(k^{2 H}+(l+1)^{2 H}\right)^{K} \\
& -\left((k+1)^{2 H}+l^{2 H}\right)^{K}+\left(k^{2 H}+l^{2 H}\right)^{K}
\end{aligned}
$$

and

$$
f_{2}(k, l)=|k-1-l|^{2 H K}+|k-l+1|^{2 H K}-2|k-l|^{2 H K} .
$$

We will obtain

$$
\mathrm{E} e^{i t T_{n}}=\mathrm{E} e^{-\frac{t^{2}}{2} \mathbf{S}_{n}},
$$

where

$$
\mathbf{S}_{n}:=\sum_{k, l=0}^{n-1} A_{k, l}^{H, K} B_{\frac{k}{n} T}^{\alpha, \beta} B_{\frac{l}{n} T}^{\alpha, \beta}
$$




$$
\begin{aligned}
& =\sum_{k, l=1}^{n-1} A_{k, l}^{H, K} B_{\frac{k}{n} T}^{\alpha, \beta} B_{\frac{l}{n} T}^{\alpha, \beta} \\
& =\sum_{k, l=1}^{n-1} A_{k, l}^{H, K}\left(\sum_{k^{\prime}=0}^{k-1}\left(B_{\frac{k^{\prime}+1}{n} T}^{\alpha, \beta}-B_{\frac{k^{\prime}}{n} T}^{\alpha, \beta}\right)\right)\left(\sum_{l^{\prime}=0}^{l-1}\left(B_{\frac{l^{\prime}+1}{n} T}^{\alpha, \beta}-B_{\frac{l^{\prime}}{n} T}^{\alpha, \beta}\right)\right) \\
& =\sum_{k^{\prime}, l^{\prime}=1}^{n-2}\left(B_{\frac{k^{\prime}+1}{n} T}^{\alpha, \beta}-B_{\frac{k^{\prime} T}{n} T}^{\alpha, \beta}\right)\left(B_{\frac{l^{\prime}+1}{n} T}^{\alpha, \beta}-B_{\frac{l^{\prime} T}{n} T}^{\alpha, \beta}\right) \sum_{l=0^{\prime}+1}^{n-1} \sum_{k=k^{\prime}+1}^{n-1} A_{k, l}^{H, K} .
\end{aligned}
$$

We first calculate

$$
\begin{aligned}
& \sum_{l=l^{\prime}+1}^{n-1} \sum_{k=k^{\prime}+1}^{n-1} A_{k, l}^{H, K} \\
= & \frac{1}{2^{K}}\left(\frac{T}{n}\right)^{2 H K} \sum_{l=l^{\prime}+1}^{n-1} \sum_{k=k^{\prime}+1}^{n-1}\left\{\left((k+1)^{2 H}+(l+1)^{2 H}\right)^{K}-\left(k^{2 H}+(l+1)^{2 H}\right)^{K}\right. \\
& -\left((k+1)^{2 H}+l^{2 H}\right)^{K}+\left(k^{2 H}+l^{2 H}\right)^{K}+\left(|k-l+1|^{2 H K}-|k-l|^{2 H K}\right) \\
& \left.-\left(|k-l|^{2 H K}-|k-l-1|^{2 H K}\right)\right\} \\
= & \frac{1}{2^{K}}\left(\frac{T}{n}\right)^{2 H K} \sum_{l=l^{\prime}+1}^{n-1}\left\{\left(n^{2 H}+(l+1)^{2 H}\right)^{K}-\left(n^{2 H}+l^{2 H}\right)^{K}-\left(\left(k^{\prime}+1\right)^{2 H}\right.\right. \\
& \left.+(l+1)^{2 H}\right)^{K}+\left(\left(k^{\prime}+1\right)^{2 H}+l^{2 H}\right)^{K}+(n-l)^{2 H K}-\left|k^{\prime}+1-l\right|^{2 H K} \\
& \left.-|n-l-1|^{2 H K}+\left|k^{\prime}-l\right|^{2 H K}\right\} \\
= & \frac{1}{2^{K}}\left(\frac{T}{n}\right)^{2 H K}\left\{\left(2 n^{2 H}\right)^{K}-\left(n^{2 H}+\left(l^{\prime}+1\right)^{2 H}\right)^{K}-\left(n^{2 H}+\left(k^{\prime}+1\right)^{2 H}\right)^{K}\right. \\
& +\left(\left(l^{\prime}+1\right)^{2 H}+\left(k^{\prime}+1\right)^{2 H}\right)^{K}+\left(n-l^{\prime}-1\right)^{2 H K}+\left(n-k^{\prime}-1\right)^{2 H K} \\
& \left.-\left|k^{\prime}-l^{\prime}\right|^{2 H K}\right\} \\
= & f^{H, K}\left(\frac{k^{\prime}+1}{n} T, \frac{l^{\prime}+1}{n} T\right),
\end{aligned}
$$

where the function $f^{H, K}(x, y)$ is given by (3.3). By combining the above calculations we get

$$
\mathbf{S}_{n}=\sum_{k, l=0}^{n-1} f^{H, K}\left(\frac{k+1}{n} T, \frac{l+1}{n} T\right)\left(B_{\frac{k+1}{n} T}^{\alpha, \beta}-B_{\frac{k}{n} T}^{\alpha, \beta}\right)\left(B_{\frac{l+1}{n} T}^{\alpha, \beta}-B_{\frac{l}{n} T}^{\alpha, \beta}\right) .
$$

Let denote $\left(\mu_{i}\right)_{i \geq 1}$ be the eigenvalues of the operator $K_{f H, K}^{\alpha, \beta}$ and by $\left(g_{i}\right)_{i \geq 1}$ the corresponding eigenfunctions. Then using Lemma 3.2, we can write

$$
f^{H, K}(x, y)=\sum_{i \geq 1} \mu_{i} g_{i}(x) g_{i}(y)
$$


with the vectors $\left(g_{i}\right)_{i \geq 1}$ orthogonal in $|\mathcal{H}|_{\alpha, \beta, S}^{2}$ and the $\mu_{i}$ are square-summable. Then the sum $\mathbf{S}_{n}$ becomes

$$
\begin{aligned}
\mathbf{S}_{n} & =\sum_{k, l=0}^{n-1}\left(\sum_{i \geq 1} \mu_{i} g_{i}\left(\frac{k+1}{n} T\right) g_{i}\left(\frac{l+1}{n} T\right)\right)\left(B_{\frac{k+1}{n} T}^{\alpha, \beta}-B_{\frac{k}{n} T}^{\alpha, \beta}\right)\left(B_{\frac{l+1}{n} T}^{\alpha, \beta}-B_{\frac{l}{n} T}^{\alpha, \beta}\right) \\
& =\sum_{i \geq 1} \mu_{i}\left(\sum_{k, l=0}^{n-1} g_{i}\left(\frac{k+1}{n} T\right)\left(B_{\frac{k+1}{n} T}^{\alpha, \beta}-B_{\frac{k}{n} T}^{\alpha, \beta}\right)\right)^{2} .
\end{aligned}
$$

Since $\alpha, \beta>\frac{1}{2}$ and $g_{i} \in|\mathcal{H}|_{\alpha, \beta, S}^{2}$, then we have that

$$
\sum_{k, l=0}^{n-1} g_{i}\left(\frac{k+1}{n} T\right)\left(B_{\frac{k+1}{n} T}^{\alpha, \beta}-B_{\frac{k}{n} T}^{\alpha, \beta}\right) \rightarrow \int_{0}^{T} g_{i}(s) d B^{\alpha, \beta}(s),
$$

in $L^{2}(\Omega)$ as $|\Delta|_{n}$ tends to zero. And therefore we have that

$$
\mathbf{S}_{n} \rightarrow \sum_{i \geq 1} \mu_{i} H_{i}^{2} \quad \text { in } \quad L^{2}(\Omega),
$$

as $n$ tends to infinity and $H_{i}=\int_{0}^{T} g_{i}(s) d B^{\alpha, \beta}(s), i \geq 1$ are independent standard normal random variables. As a consequence, since the eigenvalues are positive, then

$$
\begin{aligned}
\mathrm{E}\left(e^{i t T_{n}}\right) & =\mathrm{E}\left(\exp \left(-\frac{t^{2}}{2} \sum_{i \geq 1} \mu_{i} H_{i}^{2}\right)\right) \\
& =\prod_{i \geq 1} \mathrm{E}\left(\exp \left(-\frac{t^{2}}{2} \mu_{i} H_{i}^{2}\right)\right) \\
& =\prod_{i \geq 1}\left(\frac{1}{1+t^{2} \mu_{i}}\right)^{\frac{1}{2}} .
\end{aligned}
$$

We can state an alternative result that allows to consider the situation, when the parameters of the integrand $\alpha, \beta$ is less than $\frac{1}{2}$,

Theorem 3.2. Let $H K \in\left(\frac{1}{2}, 1\right)$ and $\alpha, \beta \in(0,1)$. Then the characteristic function of the random variable $\ell$ given by (3.1) is

$$
\mathrm{E} e^{i t \ell}=\prod_{i \geq 1}\left(1+\frac{1}{t^{2} \xi_{i}}\right)^{\frac{1}{2}},
$$

where $\left(\xi_{i}\right)_{i \geq 1}$ are the eigenvalues of the operator $K_{f^{\alpha, \beta}}^{H, K}$ given by (2.12) and $f^{\alpha, \beta}$ is defined by (3.4). 
Proof. We follow the lines of Theorem 3.1 by interchanging the roles of $X$ and $Y$ in (3.6). We obtain that

$$
\mathrm{E}\left(e^{i t \ell}\right)=\lim _{n \rightarrow \infty} \mathrm{E}\left(e^{-\frac{t^{2}}{2} \mathbf{S}_{n}}\right)
$$

where

$$
\begin{aligned}
\mathbf{S}_{n}: & =\sum_{k, l=0}^{n-1} \mathrm{E}\left(B_{\frac{k}{n} T}^{\alpha, \beta} B_{\frac{l}{n} T}^{\alpha, \beta}\right)\left(B_{\frac{k+1}{n} T}^{H, K}-B_{\frac{k}{n} T}^{H, K}\right)\left(B_{\frac{l+1}{n} T}^{H, K}-B_{\frac{l}{n} T}^{H, K}\right) \\
& =\sum_{k, l=0}^{n-1} f^{\alpha, \beta}\left(\frac{k}{n} T, \frac{l}{n} T\right)\left(B_{\frac{k+1}{n} T}^{H, K}-B_{\frac{k}{n} T}^{H, K}\right)\left(B_{\frac{l+1}{n} T}^{H, K}-B_{\frac{l}{n} T}^{H, K}\right)
\end{aligned}
$$

where $f^{\alpha, \beta}$ is given by (3.4). Now we use Lemma 3.2 and proceed as in the proof of Theorem 3.1 .

Remark 1. As a final comment, let us mote that the point 1) and 2) of Theorem 3.1 agree if $\alpha \beta$ and $H K$ are bigger than $\frac{1}{2}$. In fact it can be shown that in this case $K_{f^{\alpha, \beta}}^{H, K}$ and $K_{f H, K}^{\alpha, \beta}$ have the same eigenvalues and in this case their characteristic functions coincide term by term. Indeed, let us suppose that $\lambda \neq 0$ is an eigenvalue for $K_{f^{\alpha, \beta}, H}^{H, K}$, then their exist a non-identically zero function $\varphi_{\alpha, \beta, H, K} \in|\mathcal{H}|_{H, K}^{2}([0, T])$ such that

$$
\left(K_{f^{\alpha, \beta}}^{H, K} \varphi_{\alpha, \beta, H, K}\right)(y)=\lambda \varphi_{\alpha, \beta, H, K}(y),
$$

or

$$
\int_{0}^{T} \int_{0}^{T} R_{\alpha, \beta}(x, y) \varphi_{\alpha, \beta, H, K}\left(x^{\prime}\right) \theta^{H, K}\left(x, x^{\prime}\right) d x d x^{\prime}=\lambda \varphi_{\alpha, \beta, H, K}(y) .
$$

Let denote $\psi_{\alpha, \beta, H, K}(y)=\varphi_{\alpha, \beta, H, K}(1-y)$. It's easy to cheek that $\psi_{\alpha, \beta, H, K} \in$ $L_{\alpha, \beta}^{2}([0, T])$ and by using the change of variable $u=1-x$ and $v=1-x^{\prime}$ we obtain

$$
\left(K_{f H, K}^{\alpha, \beta} \psi_{\alpha, \beta, H, K}\right)(y)=\lambda \psi_{\alpha, \beta, H, K}(y),
$$

which implies that $\lambda$ is also an eigenvalues of $K_{f^{H, K}}^{\alpha, \beta}$.

\section{The case of two-parameter}

For any given vertors $H=\left(H_{1}, H_{2}\right) \in(0,1)^{2}$ and $K=\left(K_{1}, K_{2}\right) \in(0,1)^{2}$, an $(2,1)$-bifractional Brownian sheet $B^{H, K}=\left\{B^{H, K}(t), t \in \mathbb{R}_{+}^{2}\right\}$ is a centered Gaussian random field in $\mathbb{R}$ with i.i.d components whose covariance function are given by

$$
\begin{aligned}
R_{H, K}(t, s) & =E\left(B_{1}^{H, K}(t) B_{1}^{H, K}(s)\right) \\
& =\prod_{j=1}^{2} \frac{1}{2^{K_{j}}}\left[\left(t_{j}^{2 H_{j}}+s_{j}^{2 H_{j}}\right)^{K_{j}}-\left|t_{j}-s_{j}\right|^{2 H_{j} K_{j}}\right] .
\end{aligned}
$$


We denote $\mathcal{H}(H, K)$ the canonical Hilbert space of the Gaussian process $B_{1}^{H, K}(t)$ defined by the closure of the linear vector space generated by the elementary functions $\left\{1_{[0, t] \times[0, s]}, s, t \in[0, T]\right\}$ with respect to the inner product

$$
\left\langle 1_{[0, t]}, 1_{[0, s]}\right\rangle_{\mathcal{H}(H, K)}=R_{H, K}(t, s) .
$$

If one of $H_{i} K_{i}, i=1,2$ are greater than $\frac{1}{2}$, then the space $\mathcal{H}(H, K)$ may contains distributions. In this case, it is more convenient to work with the following set of functions $|\mathcal{H}|(H, K)$. Therefore Wiener integrals with respect to $B_{1}^{H, K}(t)$ can be naturally defined for integrands in $|\mathcal{H}|(H, K)$. The following theorem is our main result in this section.

Theorem 4.1. For $H=\left(H_{1}, H_{2}\right), K=\left(K_{1}, K_{2}\right), \alpha=\left(\alpha_{1}, \alpha_{2}\right)$ and $\beta=$ $\left(\beta_{1}, \beta_{2}\right)$. Let $H_{i} K_{i}>\frac{1}{2}, \alpha_{i} \beta_{i}>\frac{1}{2}, i=1,2$. Then the characteristic function of the random variable

$$
U=\int_{[0, T]^{2}} B_{1}^{\alpha, \beta}(t) d B_{1}^{H, K}(t)
$$

is given by

$$
E\left(e^{i t U}\right)=\prod_{j, k \geq 1} \sqrt{\frac{1}{1+t^{2} \mu_{j, 1} \mu_{k, 2}}},
$$

where $\mu_{k, r}, k \geq 1$ are the eigenvalues of the operators $K_{f^{H_{r}, K_{r}}}^{\alpha_{r}, \beta_{r}}$ with $f^{H_{r}, K_{r}}$ given by (3.3) for $r=1,2$.

Proof. The proof of the theorem is same as this of Theorem 3.1. For $t=\left(t_{1}, t_{2}\right)$ we denote by $B_{1}^{\alpha, \beta}(t)=B_{1}^{\alpha, \beta}\left(t_{1}, t_{2}\right)$. Moreover denote by

$$
A_{n}=\sum_{k_{1}, k_{2}=0}^{n-1} B_{1}^{\alpha, \beta}\left(t_{k_{1}}, t_{k_{2}}\right) B_{1}^{H, K}\left(\Delta_{k_{1}, k_{2}}\right),
$$

where

$$
\begin{aligned}
& B_{1}^{H, K}\left(\Delta_{k_{1}, k_{2}}\right) \\
= & B_{1}^{H, K}\left(t_{k_{1}+1}, t_{k_{2}+1}\right)-B_{1}^{H, K}\left(t_{k_{1}}, t_{k_{2}+1}\right)-B_{1}^{H, K}\left(t_{k_{1}+1}, t_{k_{2}}\right)+B_{1}^{H, K}\left(t_{k_{1}}, t_{k_{2}}\right),
\end{aligned}
$$

with $t_{k_{l}}=\frac{k_{l}}{n} T$ for every $k_{l}=0,1, \ldots, n-1$ and $l=1,2$. As in the proof of Lemma 3.2 , we can prove that $A_{n} \rightarrow U$ as $n$ tends to infinity in $L^{2}(\Omega)$ for $H_{r} K_{r}>\frac{1}{2}$ and $\alpha_{r} \beta_{r}>\frac{1}{2}, r=1,2$. Following the same line of reasoning as in the proof of Lemma 3.2, we have

$$
E\left(e^{i t A_{n}}\right)=E\left(e^{-\frac{1}{2} t^{2} B_{n}}\right) \quad \text { and } \quad E\left(e^{i t U}\right)=\lim _{n \rightarrow \infty} E\left(e^{-\frac{1}{2} t^{2} B_{n}}\right),
$$


where $B_{n}$ is given by

$$
\begin{aligned}
& B_{n}=\sum_{k_{1}, k_{2}=0}^{n-1} \sum_{k_{1}^{\prime}, k_{2}^{\prime}=0}^{n-1} f^{H_{1}, K_{1}}\left(\frac{k_{1}+1}{n} T, \frac{k_{1}^{\prime}+1}{n} T\right) f^{H_{2}, K_{2}}\left(\frac{k_{2}+1}{n} T, \frac{k_{2}^{\prime}+1}{n} T\right) \\
& \cdot B_{1}^{\alpha, \beta}\left(\Delta_{k_{1}, k_{2}}\right) B_{1}^{\alpha, \beta}\left(\Delta_{k_{1}^{\prime}, k_{2}^{\prime}}\right) .
\end{aligned}
$$

Let now $\mu_{k, j}, k \geq 1$ the eigenvalues of the operator $K_{f_{r} H_{r}, K_{r}}^{\alpha_{r}, \beta_{r}}, r=1,2$ and $g_{k, r}, k \geq 1, r=1,2$ the corresponding eigenfunctions. Then $\left\{g_{k, r}, k \geq 1\right\} \subset$ $|\mathcal{H}|_{\alpha_{r}, \beta_{r}}$ and the sequence $\left\{\mu_{k, r}, k \geq 1\right\}$ is square-summable for $r=1,2$, and moreover $g_{j, 1} \otimes g_{k, 2} \in|\mathcal{H}|(\alpha, \beta), j, k \geq 1$ are orthonormal, which implies that random variables

$$
B_{j, k}:=\int_{[0, T]^{2}} g_{j, 1}\left(t_{1}\right) g_{k, 2}\left(t_{2}\right) d B_{1}^{\alpha, \beta}\left(t_{1}, t_{2}\right), \quad j, k \geq 1,
$$

are independent standard normal random variables. By (1) of Lemma 3.2 it follows that

for $j=1,2$, and

$$
f^{H_{j}, K_{j}}(x, y)=\sum_{k \geq 1} \mu_{k, j} g_{k, j}(x) g_{k, j}(y)
$$

$B_{n}=\sum_{j, k \geq 1} \mu_{j, 1} \mu_{k, 2}\left(\sum_{k_{2}, k_{2}^{\prime}=0}^{n-1} g_{j, 1}\left(\frac{k_{2}+1}{n} T\right) g_{k, 2}\left(\frac{k_{2}^{\prime}+1}{n} T\right) B_{1}^{\alpha, \beta}\left(\Delta_{k_{2}, k_{2}^{\prime}}\right)\right)^{2}$.

On the other hand, it is not difficult to check that

$$
\begin{aligned}
& \sum_{k_{2}, k_{2}^{\prime}=0}^{n-1} g_{j, 1}\left(\frac{k_{2}+1}{n} T\right) g_{k, 2}\left(\frac{k_{2}^{\prime}+1}{n} T\right) B_{1}^{\alpha, \beta}\left(\Delta_{k_{2}, k_{2}^{\prime}}\right) \\
\rightarrow & \int_{0}^{T} \int_{0}^{T} g_{j, 1}(s) g_{k, 2}(t) d B_{1}^{\alpha, \beta}(s, t),
\end{aligned}
$$

in $L^{1}$, for all $j, k \geq 1$, as $n$ tends to infinity. It follows that

$$
B_{n} \longrightarrow \sum_{j, k \geq 1} \mu_{j, 1} \mu_{k, 2}\left(B_{j, k}\right)^{2}
$$

in $L^{1}$, as $n$ tends to infinity, and the theorem follows.

Theorem 4.2. Assume that $H_{r} K_{r}>\frac{1}{2}$ and $\alpha_{r} \beta_{r} \in\left(0, \frac{1}{2}\right), r=1,2$. Then

$$
E\left(e^{i t U}\right)=\prod_{j, k \geq 1} \sqrt{\frac{1}{1+t^{2} \lambda_{j, 1} \lambda_{k, 2}}},
$$

where the random variable $U$ is given by (4.3) and $\lambda_{k, r}, k \geq 1$ are the eigenvalues of the operator $K_{f^{\alpha_{r}, \beta_{r}}}^{H_{\gamma_{r}}, K_{r}}$ with $f^{\alpha_{r}, \beta_{r}}$ given by (3.4) for $r=1,2$.

Acknowledgment. The author would like to thank an anonymous referee for constructive and valuable comments and suggestions. 


\section{References}

[1] E. Alós, O. Mazet, and D. Nualart, Stochastic calculus with respect to Gaussian processes, Ann. Probab. 29 (2001), no. 2, 766-801.

[2] X. Bardina and C. A. Tudor, The law of a stochastic integral with two independent fractional Brownian motions, Bol. Soc. Mat. Mexicana (3) 13 (2007), no. 1, 231-242.

[3] R. Berthuet, Loi du logharitme itéré pour cetaines intégrales stochastiques, Ann. Sci. Univ. Clérmont-Ferrand Math. 69 (1981), 9-18.

[4] F. Biagini, Y. Hu, B. Øksendal, and T. Zhang, Stochastic Calculus for Fractional Brownian Motion and Applications, Springer-Verlag London, Ltd., London, 2008.

[5] P. Caithamer, Decoupled double stochastic fractional integrals, Stochastics 77 (2005), no. 3, 205-210.

[6] K. Es-Sebaiy and C. A. Tudor, Multidimensional bifractional Brownian motion: Itô and Tanaka formulas, Stoch. Dyn. 7 (2007), no. 3, 365-388.

[7] C. Houdré and J. Villa, An example of infinite dimensional quasi-helix, Stochastic models (Mexico City, 2002), 195-201, Contemp. Math., 336, Amer. Math. Soc., Providence, RI, 2003.

[8] Y. Hu, Integral transformations and anticipative calculus for fractional Brownian motions, Mem. Amer. Math. Soc. 175 (2005), no. 825, viii+127 pp.

[9] R. Klein and E. Giné, On quadratic variation of processes with Gaussian increments, Ann. Probab. 3 (1975), no. 4, 716-721.

[10] I. Kruk, F. Russo, and C. A. Tudor, Wiener integrals, Malliavin calculus and covariance measure structure, J. Funct. Anal. 249 (2007), no. 1, 92-142.

[11] P. Lei and D. Nualart, A decomposition of the bifractional Brownian motion and some applications, Statist. Probab. Lett. 79 (2009), no. 5, 619-624.

[12] P. Lévy, Wiener's random function, and other Laplacian random functions, Proceedings of the Second Berkeley Symposium on Mathematical Statistics and Probability, 1950, pp. 171-187. University of California Press, Berkeley and Los Angeles, 1951.

[13] Y. Mishura, Stochastic Calculus for Fractional Brownian Motion and Related Processes, Lecture Notes in Mathematics, 1929. Springer-Verlag, Berlin, 2008.

[14] D. Nualart, Malliavin Calculus and Related Topics, 2nd edition Springer, New York, 2006.

[15] P. E. Protter, Stochastic Integration and Differential Equations, Springer-Verlag, Berlin, 2005.

[16] F. Russo and C. A. Tudor, On bifractional Brownian motion, Stochastic Process. Appl. 116 (2006), no. 5, 830-856.

[17] C. A. Tudor and Y. Xiao, Sample path properties of bifractional Brownian motion, Bernoulli 13 (2007), no. 4, 1023-1052.

[18] L. Yan, J. Liu, and G. Jing, Quadratic covariation and Itô formula for a bifractional Brownian motion, preprint (2008).

[19] M. Yor, Remarques sur une formule de Paul Lévy, pp. 343-346, Lecture Notes in Math., 784, Springer, Berlin, 1980.

School of Mathematics and Statistics

Nanjing Audit University

86 West Yushan Rd., Pokou

NANJing 211815, P. R. China

E-mail address: jordanjunfeng@163.com 